\section{The Analgesic and Anti-Stress Effects of a Kampo Medicine (Yokukansan) in Rats with Chronic Constriction Injury - A Comparative Study with Kamishoyosan}

Keywords: Yokukansan; Kamishoyosan; Kampo medicine; Analgesic effect; Anti-stress effect; Neuropathic pain model rat

\begin{abstract}
Background: Yokukansan (YKS), which is a Japanese traditional herbal (Kampo) medicine, is composed of seven crude drugs: Uncariae cum uncis ramulus, Cnidii rhizoma, Bupleuri radix, Atractylodis lanceae rhizoma, Poria, Angelicae radix and Glycyrrhizae radix. Although YKS is typically administered to patients with symptoms such as emotional irritability and neurosis, it has also recently been reported to be effective against pain disorders. Kamishoyosan (KSS), which contains similar component galenicals to YKS, is used to treat neuropsychiatric symptoms. The present study investigated the effects of these medicines on neuropathic pain and the stress caused by pain.

Methods: The present study used a rat model of chronic constriction injury $(\mathrm{CCl})$. At week 2 post- $\mathrm{CCl}$, decreases in the mechanical and thermal withdrawal thresholds were confirmed in the $\mathrm{CCl}$ rats and the drugs were administered for a two-week period. At week 4 post-CCl, the levels of plasma corticosterone and chromogranin A, which are markers of mental stress, were measured to evaluate the anti-stress effects. Furthermore, we investigated the changes in pain threshold and spinal astrocytes, which are involved in the expression of chronic pain, to evaluate the analgesic effects.
\end{abstract}

Results: The levels of plasma corticosterone and chromogranin A significantly increased in the $\mathrm{CCl}$ rats. Moreover, the pain threshold significantly decreased and the activation of the spinal astrocytes was observed. The increases in the levels of corticosterone and chromogranin A were significantly suppressed by the administration of either YKS or KSS. However, the control of astrocytic activation and the decrease in the pain threshold only occurred after the administration of YKS.

Conclusion: The results indicate that YKS effectively reduces both the stress caused by pain and neuropathic pain.

\section{Abbreviations}

CCI: Chronic Constriction Injury; GFAP: Glial Fibrillary Acidic Protein; HPA: Hypothalamic-Pituitary-Adrenal; KSS: Kamishoyosan; YKS: Yokukansan

\section{Introduction}

Chronic pain causes stress and psychiatric symptoms, such as anxiety and depression. It is therefore necessary to provide mental health care in association with pain management. Yokukansan (YKS), which is a Japanese traditional herbal (Kampo) medicine
Journal of

Integrative Medicine \& Therapy

\section{Hiroki Suga ${ }^{1,2}$, Masataka Sunagawa ${ }^{1 *}$, Hideshi Ikemoto', Takako Nakanishi' ${ }^{1}$, Aki Fujiwara1, Mayumi Okada ${ }^{3}$, Hitoshi Mera ${ }^{2}$, Shogo Ishino ${ }^{1}$ and Tadashi Hisamitsu ${ }^{1}$}

${ }^{I}$ Department of Physiology, School of Medicine, Showa University, Tokyo, Japan

${ }^{2}$ Department of Anesthesiology, Tokyo Metropolitan Health and Medical Corporation Ebara Hospital, Tokyo, Japan

${ }^{3}$ Department of Anesthesiology, School of Medicine, Showa University, Tokyo, Japan

\section{*Address for Correspondence}

Masataka Sunagawa, Department of Physiology, School of Medicine, Showa University, Tokyo 142-8555, Japan, Tel: +81-3-3784-8110; Fax: +81-3-3784-5368; E-mail: suna@med.showa-u.ac.jp

\section{Submission: 04 November 2015}

Accepted: 15 December 2015

Published: 21 December 2015

Copyright: $\odot 2015$ Suga H, et al. This is an open access article distributed under the Creative Commons Attribution License, which permits unrestricted use, distribution, and reproduction in any medium, provided the original work is properly cited.

Reviewed \& Approved by: Dr. Harold H. Fain, Assistant Professor of Community Medicine, University of North Texas Health Science Center, USA

(Table 1), has been administered to patients who show symptoms such as emotional irritability, neurosis and insomnia [1]. YKS has recently been reported to be effective against pain disorders, such as headache and chronic pain $[2,3]$. Several studies have recently been performed to clarify the pharmacological actions of YKS. In the glutamatergic neuronal system, YKS was demonstrated to have an ameliorative effect on glutamate clearance in astrocytes [4] and to have an antagonistic action at the NMDA receptor [5]. In the serotonin-responsive nervous system, YKS has a partial agonistic effect on the serotonin $1 \mathrm{~A}$ receptor, which is associated with analgesia [6] and a downregulatory effect on the serotonin $2 \mathrm{~A}$ receptor, which is associated with pain [7].

We have previously reported that YKS had analgesic and antistress effects in a rat model of chronic inflammatory chronic pain [8]. In this study, we investigated the efficacy of YKS against neuropathic pain. Neuropathic pain is defined as pain which is directly caused by somatosensory injury or disease, and which is associated with symptoms such as spontaneous pain, allodynia and hyperalgesia [9]. The drug therapy used for neuropathic pain primarily includes tricyclic antidepressants, calcium channel $\alpha 2 \delta$ ligands, anti-arrhythmic drugs, serotonin noradrenaline reuptake inhibitors, anti-epileptic drugs and narcotic analgesics [10]. However, in many cases, these drugs do not sufficiently treat the symptoms, and some patients are not able to take the drugs continuously because of their side effects.

In the present study, we used a rat model of chronic constriction injury (CCI), which was prepared according to the model described 
Citation: Suga H, Sunagawa M, Ikemoto H, Nakanishi T, Fujiwara A, et al. The Analgesic and Anti-Stress Effects of a Kampo Medicine (Yokukansan) in Rats with Chronic Constriction Injury - A Comparative Study with Kamishoyosan. J Integrative Med Ther. 2015;2(2): 5.

ISSN: $2378-1343$

Table 1: The components of Yokukansan (YKS; TJ-54) and Kamishoyosan (KSS; TJ-24). The weights show the mixing ratio.

\begin{tabular}{|l|c|c|}
\hline & $\begin{array}{c}\text { Yokukansan } \\
\text { (YKS) }\end{array}$ & $\begin{array}{c}\text { Kamishoyosan } \\
\text { (KSS) }\end{array}$ \\
\hline Uncariae cum uncis ramulus & $3.0 \mathrm{~g}$ & \\
\hline Cnidii rhizoma & $3.0 \mathrm{~g}$ & $3.0 \mathrm{~g}$ \\
\hline Bupleuri radix & $2.0 \mathrm{~g}$ & $3.0 \mathrm{~g}$ \\
\hline Atratylodis lanceae rhizoma & $4.0 \mathrm{~g}$ & $3.0 \mathrm{~g}$ \\
\hline Poria & $4.0 \mathrm{~g}$ & $3.0 \mathrm{~g}$ \\
\hline Angelicae radix & $3.0 \mathrm{~g}$ & $1.5 \mathrm{~g}$ \\
\hline Glycyrrhizae radix & $1.5 \mathrm{~g}$ & $3.0 \mathrm{~g}$ \\
\hline Paeoniae radix & & $2.0 \mathrm{~g}$ \\
\hline Gardeniae fructus & & $2.0 \mathrm{~g}$ \\
\hline Moutan cortex & & $1.0 \mathrm{~g}$ \\
\hline Zingiberis rhizoma & & $1.0 \mathrm{~g}$ \\
\hline Menthae herba & & \\
\hline
\end{tabular}

by Bennett, to investigate the effects of YKS against pain and the stress that accompanied the pain in comparison to Kamishoyosan (KSS) (Table 1), another Kampo medicine which contains component galenicals which are similar to YKS, and which is used to treat neuropsychiatric symptoms such as anxiety, insomnia and irritability [11,12]. We also investigated the effects of these treatments on astrocytes in the spinal cord to study the mechanism of action underlying the analgesic effect. It has been reported that astrocytes, one of the glial cells in the central nervous system, may play an important role in the expression of neuropathic pain. Increases in astrocytes and astrogliosis (hypertrophy and proliferation of astrocytes) are observed in the spinal cords of rats with peripheral nerve injury, suggesting that pain is a systemic reaction $[13,14]$.

\section{Materials and Methods}

\section{Animals}

Thirty-two male Wistar rats (eight weeks old, weighing 250 - 300 g), which were purchased from Nippon Bio-Supp. Center (Tokyo, Japan), were randomly divided into four groups (eight per group): sham control rats (Control), CCI model rats (CCI), YKS-treated CCI model rats (YKS) and KSS-treated CCI model rats (KSS). During the experiment period, the animals were housed in standard plastic cages, and were kept in our animal facilities at $25 \pm 2{ }^{\circ} \mathrm{C}$ in $55 \pm 5 \%$ humidity in a 12/h light/dark cycle. Food and water were provided ad libitum. All experiments were carried out according to the guidelines of the Committee of Animal Care and Welfare of Showa University (certificate number: 03061). The model of CCI of the sciatic nerve was created according to the methods described by Bennett and Xie [11]. This model is commonly used for the study of neuropathic pain and its treatment and shows many of the pathophysiological properties of chronic neuropathic pain, such as allodynia and hyperalgesia. These manifestations appear 48 hours after CCI and last for more than two months [11]. Rats were intraperitoneally anesthetized with pentobarbital sodium $\left(50 \mathrm{mg} / \mathrm{kg}\right.$ ) (Somnopentyl ${ }^{\oplus}$, Kyoritsu Seiyaku Co., Tokyo, Japan), and a 10-mm segment of the left common sciatic nerve was exposed at the mid-thigh level. Four ligatures (4-0 silk thread), with about 1-mm spacing, were loosely tied around the nerve. The operation was completed by closing the muscles and skin.
The sham operation was performed without nerve ligation.

\section{Administration of Kampo medicines}

YKS (Lot No. 2110054010) or KSS (Lot No. 2100024010) (manufactured by Tsumura \& Co., Tokyo, Japan) (Table 1) was mixed with powdered rodent chow (CE-2: CLEA Japan, Inc., Tokyo, Japan) at a concentration of $3 \%$. The rats were fed with this chow from two weeks after the CCI. The rats in the Control and CCI groups were fed powdered chow without YKS or KSS. The concentration was chosen by referring to the effective doses of YKS [8] or other Kampo medicines [15] in previous reports. Body weight was measured weekly to examine the influence of YKS and KSS on appetite, nutrient absorption and metabolism.

\section{Pain threshold}

Mechanical hyperalgesia was assessed by measuring the withdrawal threshold of the left hind paw in response to a mechanical stimulus using an electronic von Frey anesthesiometer (model 2390; IITC Life Science, CA, USA) [16]. Each animal was placed in a metal mesh cage raised $25 \mathrm{~cm}$ above the floor. The animals were allowed to acclimate to the environment for $10 \mathrm{~min}$. A rigid tip attached to the meter was applied to the left plantar surface from under the floor. Thermal hyperalgesia was assessed by measuring the hind paw withdrawal latency in response to radiant heat using a plantar test apparatus (model 37370; Ugo Basile, Comerio, Italy) according to the method described by Hargreaves et al. [17]. Each rat was placed into a compartment enclosure on a glass surface. A beam of infrared radiation was directed upward through a glass plate on which the rat was placed, heating the skin of the hind paw.

The mean withdrawal latency of each measurement was determined from the average of three trials separated by a 5 -min interval. Tests were performed before the CCI procedure and weeks 2,3 and 4 post-CCI.

\section{Immunohistochemistry}

At week 4 post-CCI, the rats were intraperitoneally anesthetized with pentobarbital sodium $(50 \mathrm{mg} / \mathrm{kg})$ and intracardially perfused with phosphate buffered saline at $\mathrm{pH} 7.4$ (PBS) until all of the blood had been removed from the system. After perfusion with $4 \%$ paraformaldehyde in $0.1 \mathrm{M}$ PBS, the fourth lumbar spinal cords (L4) were harvested. Increasing evidence has shown that astrocytes play important roles in the development of chronic pain $[13,14]$. The appearance of activated astrocytes in the L4 extracted from sixteen rats (four per group) was investigated by an immunohistochemical analysis. Glial fibrillary acidic protein (GFAP) staining was performed to label the activated astrocytes, because the protein is expressed exclusively in astrocytes and because astroglial activation causes the increased expression of GFAP and astrogliosis [13,14]. Tissue specimens were embedded in OTC compound, frozen and then cut into $10 \mu \mathrm{m}$ sections with a cryostat. All of the sections were incubated overnight at $4{ }^{\circ} \mathrm{C}$ with mouse anti-GFAP-Cy3 antibody (C9205; 1:500, Sigma-Aldrich, St. Louis, MO, USA). Samples were observed by a confocal laser scanning fluorescence microscope (FV1000D, Olympus Corp., Tokyo, Japan) and the expression of GFAP was analyzed and quantified using the Image J-software program (http:// rsb.info.nih.gov). The values were expressed as the area fraction, which is the ratio of the area with expression of GFAP within a demarcated region $(300 \mu \mathrm{m} \times 300 \mu \mathrm{m})$ in the spinal dorsal horn. All 
Citation: Suga H, Sunagawa M, Ikemoto H, Nakanishi T, Fujiwara A, et al. The Analgesic and Anti-Stress Effects of a Kampo Medicine (Yokukansan) in Rats with Chronic Constriction Injury - A Comparative Study with Kamishoyosan. J Integrative Med Ther. 2015;2(2): 5.

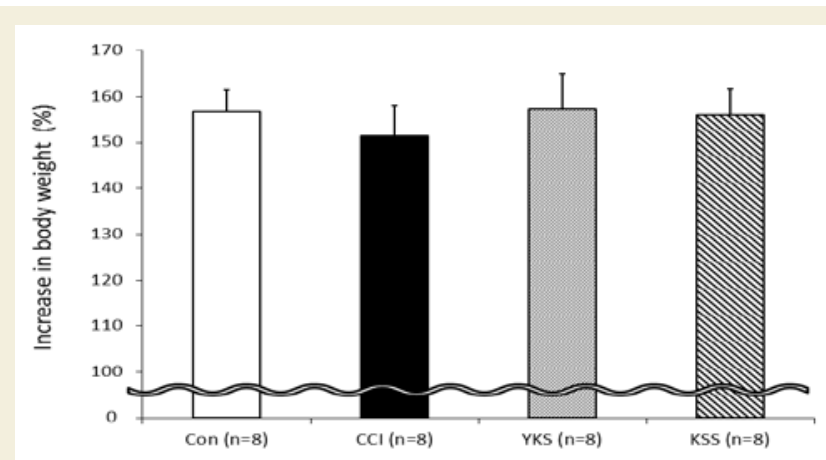

Figure 1: The body weight increases in the different groups. The weight gain at week 4 showed no significant differences in comparison to baseline among the four groups. The data are expressed as the means \pm SEM (Con, sham control rats; $\mathrm{CCI}, \mathrm{CCl}$ model rats; YKS, Yokukansan-treated $\mathrm{CCI}$ model rats; KSS, Kamishoyosan-treated $\mathrm{CCl}$ model rats).

values were reported as an average of at least ten micrographs per rat.

\section{Estimation of stress}

At week 4 post-CCI, the rats were intraperitoneally anesthetized with pentobarbital sodium $(50 \mathrm{mg} / \mathrm{kg})$ and blood samples were taken from the inferior vena cava. The levels of plasma corticosterone and chromogranin A (CgA) in the thirty-two rats (eight per group) were measured using ELISA kits (corticosterone: ADI-900-097; Enzo Life Sciences, Farmingdale, NY) (CgA: YK070; Yanaihara Institute Inc., Shizuoka, Japan) to evaluate their physiological stress [18].

\section{Statistical analysis}

The experimental data are shown as the means \pm standard errors. The post-hoc Tukey test was used for the statistical analysis. $P$ values of $<0.05$ were considered to be statistically significant.

\section{Results}

\section{Changes in body weight}

Body weight was measured every week during the experimental period. At week 4 , none of the groups showed a significant increase in body weight from baseline (Con: $156.67 \pm 4.73 \%$, CCI: $151.46 \pm 6.42 \%$, YKS: $157.19 \pm 7.82 \%$, KSS: $155.86 \pm 5.72 \%$ ) (eight per group) (Figure 1). YKS and KSS may have no influence on appetite, nutrient absorption or metabolism.

\section{Analgesic effect}

Mechanical and thermal hyperalgesia was assessed by measuring the withdrawal threshold (eight per group) (Figure 2). At week 2, the withdrawal thresholds of the hind paw in response to mechanical and thermal stimuli were decreased in the rats that underwent CCI surgery. From week 2 post-CCI, the herbal medicines were administered for two weeks. At week 4 post-CCI, the mechanical withdrawal latency in the CCI group was significantly reduced $(18.77 \pm 3.97 \mathrm{~g})$, but this decrease was significantly inhibited in the YKS group $(28.82 \pm 4.50$ g) $(\mathrm{P}<0.05$ compared with the CCI group) (Figure $2 \mathrm{~A})$. Similarly, the thermal withdrawal latency in the CCI group was significantly reduced $(2.96 \pm 0.60 \mathrm{sec})$, but this decrease was significantly inhibited in the YKS group $(5.15 \pm 1.05 \mathrm{sec})(\mathrm{P}<0.05$ compared with the CCI group) (Figure $2 \mathrm{~B}$ ). The administration of YKS therefore inhibited the CCI-induced mechanical and thermal hyperalgesia.

\section{The inhibition of astrocytic activation}

The GFAP immunoreactivity was assessed in the spinal cord (L4) at week 4 post-CCI (four per group) (Figure 3 ). In the CCI group, the number of GFAP $(+)$ cells in the ipsilateral lumbar dorsal horn was increased (Figure $3 \mathrm{~B}$ ) and the cells were hypertrophied (Figure $3 \mathrm{~F}$ ). Although these changes were not suppressed by the administration of KSS (Figure 3D and $\mathrm{H}$ ), they were inhibited by the administration of YKS (Figure 3C and G).

The GFAP value was then expressed as an area fraction on images (magnification; $\times 200$ ) (Figure 3I). In the CCI group, the GFAP level $(12.90 \pm 3.16 \%)$ was significantly increased in comparison to the Control group $(4.66 \pm 1.28 \%)(\mathrm{P}<0.05)$. This increase was significantly inhibited by the administration of YKS $(4.98 \pm 2.54 \%)(\mathrm{P}<0.05)$. The administration of KSS $(8.25 \pm 1.69 \%)$ had no significant effect on astrocytic activation.

\section{Anti-stress effect}

The levels of plasma corticosterone and CgA were measured to examine whether the administration of the medications affected the level of stress caused by chronic pain ( $\mathrm{n}=8 /$ group) (Figure 4$)$. The corticosterone level is used as a marker of endocrine system activity, and the CgA level is used as a marker of sympathetic activity [18]. At week 4 post-CCI, the levels of corticosterone $(244.36 \pm 35.77 \mathrm{ng} /$ $\mathrm{ml})$ and $\mathrm{CgA}(29.65 \pm 6.22 \mathrm{ng} / \mathrm{ml})$ in the CCI group were significantly increased in comparison to the Control group (corticosterone; $87.08 \pm 6.67 \mathrm{ng} / \mathrm{ml}, \mathrm{CgA} ; 15.02 \pm 1.00 \mathrm{ng} / \mathrm{ml})(\mathrm{P}<0.01)$. However, the increases were significantly inhibited by the administration of YKS (corticosterone; $110.67 \pm 17.41 \mathrm{ng} / \mathrm{ml}, \mathrm{CgA} ; 15.07 \pm 1.28 \mathrm{ng} / \mathrm{ml}$ ) and KSS (corticosterone; $115.42 \pm 20.19 \mathrm{ng} / \mathrm{ml}, \mathrm{CgA} ; 15.43 \pm 2.11 \mathrm{ng} / \mathrm{ml}$ ).

\section{Discussion}

Several recent clinical reports have indicated that YKS is effective against neuropathic pain, such as post-herpetic neuralgia, central pain, complex regional pain syndrome (CRPS) and trigeminal neuralgia $[2,3]$. In an animal study, Suzuki et al. reported that a single administration of YKS inhibited mechanical and cold allodynia for

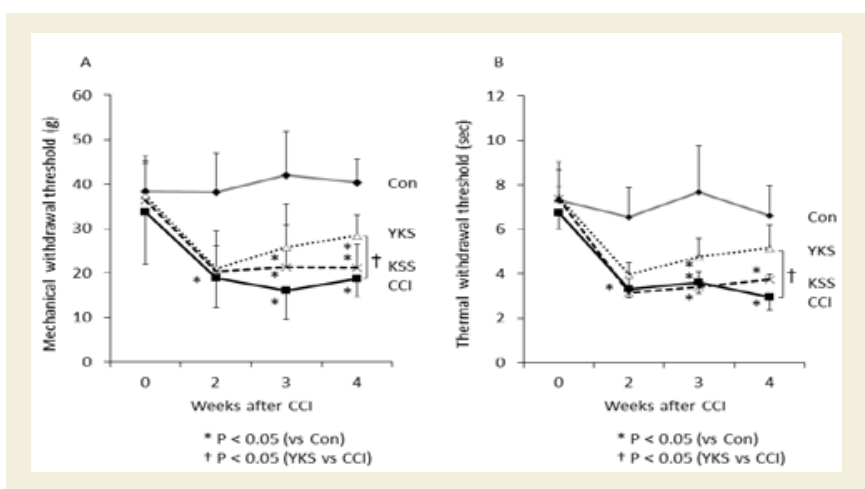

Figure 2: The effects of Yokukansan and Kamishoyosan on $\mathrm{CCl}$-induced mechanical (A) and thermal (B) hyperalgesia. The values at week 2 in the $\mathrm{CCI}, \mathrm{YKS}$ and $\mathrm{KSS}$ groups were significantly decreased in comparison to the control group. At week 4, the values in the YKS group were increased in comparison to the $\mathrm{CCl}$ group $(\dagger \mathrm{P}<0.05)$. The data are expressed as the means \pm SEM. 

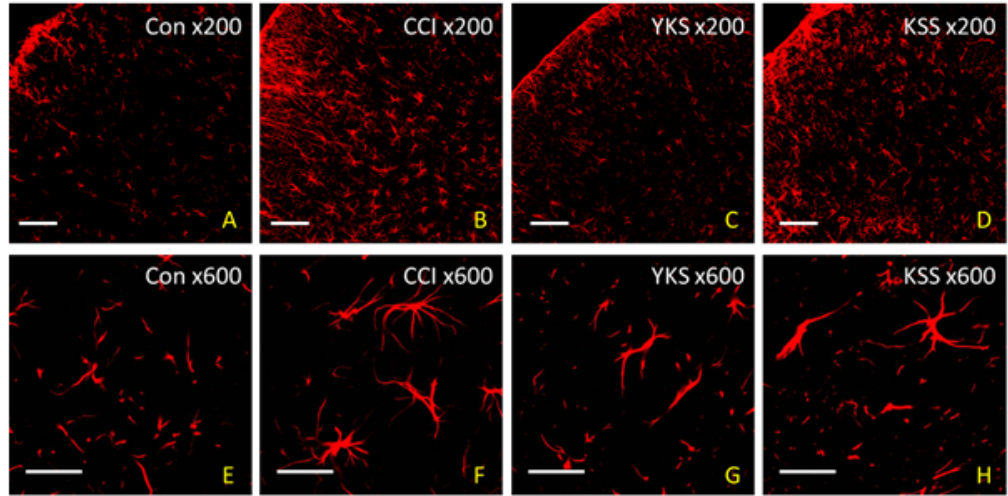

I

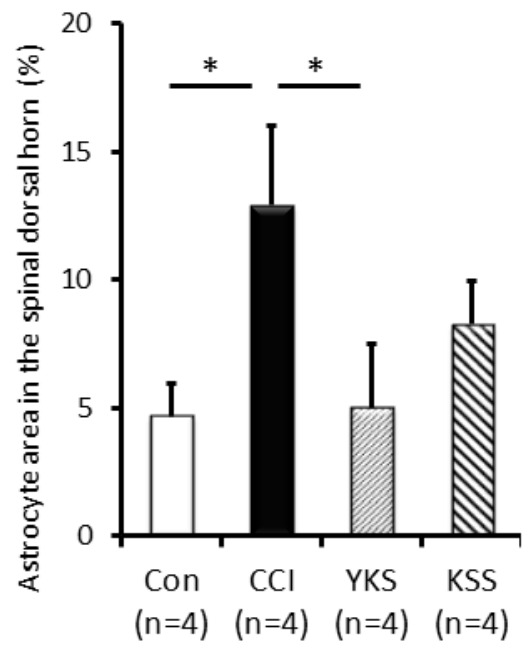

$* P<0.05$

Figure 3: The effects of Yokukansan on the CCl-induced increase in GFAP immunoreactivity in the spinal cord (L4). (A)-(D) Low magnification images (x200) of the L4 spinal cord. Scale bar=100 $\mu \mathrm{m}$. The number of GFAP (+) cells in the CCl group was clearly increased in comparison to the control group. The increase was obviously smaller in the YKS group, but there was no clear inhibition of the increase in the KSS group. (E)-(H) High magnification images (x600) of the L4 spinal dorsal horn. Scale bar $=50 \mu \mathrm{m}$. The GFAP $(+)$ cells in the $\mathrm{CCl}$ group were clearly hypertrophied, while this change was prevented in the YKS group. The change was not clearly inhibited in the KSS group. (I) The GFAP value is expressed as a fraction of the area on the images (magnification: $\times 200$ ). The CCI group showed a significant increase in the GFAP level in comparison to the Control group. This increase was significantly inhibited in the YKS group, but not in the KSS group $\left({ }^{*} \mathrm{P}<0.05\right)$. The data are expressed as the means \pm SEM.

a few hours after the administration using the same CCI model as the present study [19]. Our study suggests that the continuous administration of YKS is effective for retaining an analgesic effect (Figure 2).

The present study showed that YKS, but not KSS, had an analgesic effect (Figures 2 and 3). The principal components of the two herbal medicines are Bupleuri radix, Angelicae radix, Glycyrrhizae radix, Atratylodis lanceae rhizoma and Poria, with YKS also containing Uncariae cum Uncis ramulus and Cnidii rhizoma (Table 1). Uncariae cum Uncis ramulus has been demonstrated to have several pharmacological actions, including serotonin 1A receptor stimulation, serotonin $2 \mathrm{~A}$ receptor antagonism and an inhibitory action against the NMDA receptor-mediated ion current [5]. In the descending inhibitory serotonergic system, serotonin exerts an analgesic effect by inhibiting the release of glutamate, an excitatory neurotransmitter, from primary afferent fibers in the surface of the lumbar dorsal horn via the serotonin $1 \mathrm{~A}$ receptors [20]. Furthermore, the administration of serotonin $2 \mathrm{~A}$ receptor antagonists reduced the hyperalgesia induced in a rat model of acute inflammation [21].

On the other hand, tetramethylpyrazine, an alkaloid present in Cnidii rhizomawas shown to have an analgesic effect through the inhibition of neuronal apoptosis and the control of the $\mathrm{P} 2 \mathrm{X} 3$ receptor, which is involved in the development of neuropathic pain [22]. These pharmacological effects of Uncariae cum Uncis ramulus and Cnidii rhizoma may play an important role in the analgesic effects of YKS.

In recent years, it has been hypothesized that activated microglial cells are involved in the appearance of chronic pain, while activated astrocytes are involved in the maintenance of chronic pain. Microglial activation was seen on day 3 after nerve injury and peaked on day 14 . On the other hand, the astroglial activation after nerve injury is more persistent than the microglial reaction. It has been reported that the reaction begins on day 7 and lasts for more than 150 days after nerve injury [23]. In the present study, the quantitative and morphological changes of astrocytes in the CCI group were investigated by immunohistochemistry (Figure 3). Although the inhibitory effects of YKS on the activation of microglial cells have previously been reported $[8,24]$, YKS may also has inhibitory effects on the activation of astrocytes. The analgesic effect of YKS on neuropathic pain may therefore be provided through the inhibition of both microglial and astrocytic activation.

YKS has traditionally been administered to patients who show symptoms such as emotional irritability, agitation and neurosis [1]. Thus, in the present study, we also investigated whether YKS exhibited any effects against the stress caused by chronic pain (Figure 4). The corticosterone level is commonly used as a marker of the hypothalamic-pituitary-adrenal (HPA) axis activity, while the CgA level is used as a marker of sympathetic activity [18]. The levels of these markers were increased significantly in the CCI group. However, these increases were significantly inhibited by the administration of either YKS or KSS. This result suggests that both YKS and KSS have inhibitory effects on HPA axis hyperactivity and the sympathetic arousal caused by pain stress.

In a previous report using experimental animal models, Glycyrrhizae radix and Bupleuri radix (which are contained in YKS and KSS) were shown to exert anti-stress effects [25,26]. Regarding the potential mechanism of action of YKS, the expression of the serotonin $2 \mathrm{~A}$ receptor in the prefrontal cortex, which is involved in the induction of anxiety, was found to be decreased [7]. Furthermore, it was previously shown that the geissoschizine methyl ether isolated from Uncariae cum Uncis ramulus has anxiolytic effects through its 
Citation: Suga H, Sunagawa M, Ikemoto H, Nakanishi T, Fujiwara A, et al. The Analgesic and Anti-Stress Effects of a Kampo Medicine (Yokukansan) in Rats with Chronic Constriction Injury - A Comparative Study with Kamishoyosan. J Integrative Med Ther. 2015;2(2): 5.
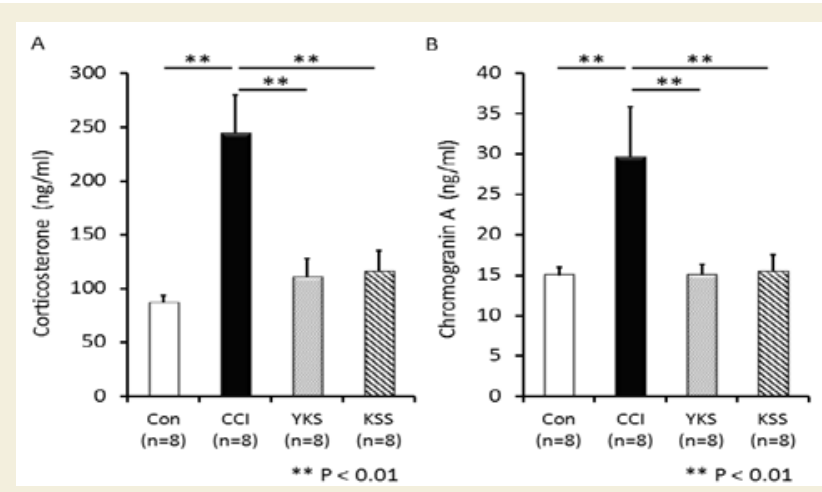

Figure 4: The effects of Yokukansan and Kamishoyosan on the levels of plasma corticosterone (A) and chromogranin A (B). At week 4 post-CCl, the levels of plasma corticosterone and chromogranin A were significantly increased in the $\mathrm{CCl}$ group in comparison to the control group. These increases were significantly suppressed in the YKS and KSS groups $\left({ }^{* *} \mathrm{P}<0.01\right)$. The data are expressed as the means \pm SEM.

action on the serotonin $1 \mathrm{~A}$ receptor [27].

Taken together, the findings of the present study indicate that YKS reduces both neuropathic pain and the stress caused by pain. Given that YKS had similar effects in rat models of chronic inflammatory pain [8]; it is possible that YKS may be widely applied to the treatment of patients with various types of chronic pain.

\section{Conclusions}

YKS reduced the chronic neuropathic pain and the stress caused by pain in a rat model of CCI, and was more effective than KSS, which only reduced the stress associated with chronic pain.

\section{References}

1. de Caires S, Steenkamp V (2010) Use of Yokukansan (TJ-54) in the treatment of neurological disorders: a review. Phytother Res 24: 1265-1270.

2. Nakamura Y, Tajima K, Kawagoe I, Kanai M, Mitsuhata H (2009) Efficacy of traditional herbal medicine, Yokukansan on patients with neuropathic pain. Masui 58: 1248-1255.

3. Yamaguchi K (2015) Traditional Japanese herbal medicines for treatment of odontopathy. Front Pharmacol 6: 176.

4. Takeda A, Itoh H, Tamano H, Yuzurihara M, Oku N (2008) Suppressive effect of Yokukansan on excessive release of glutamate and aspartate in the hippocampus of zinc-deficient rats. Nutr Neurosci 11: 41-46.

5. Kawakami Z, Ikarashi $Y$, Kase $Y$ (2011) Isoliquiritigenin is a novel NMDA receptor antagonist in kampo medicine yokukansan. Cell Mol Neurobiol 31: 1203-1212.

6. Terawaki K, Ikarashi Y, Sekiguchi K, Nakai Y, Kase Y (2010) Partial agonistic effect of yokukansan on human recombinant serotonin $1 \mathrm{~A}$ receptors expressed in the membranes of Chinese hamster ovary cells. J Ethnopharmacol 127: 306-312.

7. Egashira N, Iwasaki K, Ishibashi A, Hayakawa K, Okuno R, et al. (2008) Repeated administration of Yokukansan inhibits DOI-induced head-twitch response and decreases expression of 5-hydroxytryptamine (5-HT)2A receptors in the prefrontal cortex. Prog Neuropsychopharmacol Biol Psychiatry 32: 1516-1520.

8. Honda $\mathrm{Y}$, Sunagawa M, Yoneyama S, Ikemoto $\mathrm{H}$, Nakanishi-Ueda $\mathrm{T}$, et al (2012) Analgesic and anti-stress effects of Yokukansan in rats with adjuvant arthritis. Kampo Med 64: 78-85.

9. Woolf CJ, Mannion RJ (1999) Neuropathic pain: aetiology, symptoms, mechanisms, and management. Lancet 353: 1959-1964.
10. Dworkin RH, O'Connor AB, Backonja M, Farrar JT, Finnerup NB, et al. (2007) Pharmacologic management of neuropathic pain: evidence-based recommendations. Pain 132: 237-251.

11. Bennett GJ, Xie YK (1988) A peripheral mononeuropathy in rat that produces disorders of pain sensation like those seen in man. Pain 33: 87-107.

12. Yamada K, Kanba S (2007) Effectiveness of kamishoyosan for premenstrual dysphoric disorder: open-labeled pilot study. Psychiatry Clin Neurosci 61: 323-325.

13. Gao YJ, Ji RR (2010) Targeting astrocyte signaling for chronic pain. Neurotherapeutics 7: 482-493.

14. de Novellis V, Siniscalco D, Galderisi U, Fuccio C, Nolano M, et al. (2004) Blockade of glutamate mGlu5 receptors in a rat model of neuropathic pain prevents early over-expression of pro-apoptotic genes and morphological changes in dorsal horn lamina II. Neuropharmacology 46: 468-479.

15. Goto M, Hayashi M, Todoroki T, Seyama Y, Yamashita S (1992) Effects of traditional Chinese medicines (dai-saiko-to, sho-saiko-to and hachimi-ziogan) on spontaneously diabetic rat (WBN/Kob) with experimentally induced lipid and mineral disorders. Nihon Yakurigaku Zasshi 100: 353-358.

16. Ambalavanar R, Moritani M, Moutanni A, Gangula $P$, Yallampalli $C$, et al. (2006) Deep tissue inflammation upregulates neuropeptides and evokes nociceptive behaviors which are modulated by a neuropeptide antagonist. Pain 120: 53-68.

17. Hargreaves K, Dubner R, Brown F, Flores C, Joris J (1988) A new and sensitive method for measuring thermal nociception in cutaneous hyperalgesia. Pain 32: 77-88.

18. Lindahl AE, Low A, Stridsberg M, Sjöberg F, Ekselius L, et al. (2013) Plasma chromogranin A after severe burn trauma. Neuropeptides 47: 207-212.

19. Suzuki Y, Mitsuhata H, Yuzurihara M, Kase $Y$ (2012) Antiallodynic effect of herbal medicine yokukansan on peripheral neuropathy in rats with chronic constriction injury. Evid Based Complement Alternat Med 2012: 953459.

20. Ito A, Kumamoto E, Takeda M, Shibata K, Sagai H, et al. (2000) Mechanisms for ovariectomy-induced hyperalgesia and its relief by calcitonin: participation of 5-HT1A-like receptor on C-afferent terminals in substantia gelatinosa of the rat spinal cord. J Neurosci 20: 6302-6308.

21. Abbott FV, Hong $Y$, Blier P (1996) Activation of 5-HT2A receptors potentiates pain produced by inflammatory mediators. Neuropharmacology 35: 99-110.

22. Leng YF, Gao XM, Wang SX, Xing YH (2012) Effects of tetramethylpyrazine on neuronal apoptosis in the superficial dorsal horn in a rat model of neuropathic pain. Am J Chin Med 40: 1229-1239.

23. Zhang J, De Koninck $Y$ (2006) Spatial and temporal relationship between monocyte chemoattractant protein-1 expression and spinal glial activation following peripheral nerve injury. J Neurochem 97: 772-783.

24. Furuya M, Miyaoka T, Tsumori T, Liaury K, Hashioka S, et al. (2013) Yokukansan promotes hippocampal neurogenesis associated with the suppression of activated microglia in Gunn rat. J Neuroinflammation 10: 145.

25. Park HJ, Shim HS, Kim H, Kim KS, Lee H, et al. (2010) Effects of Glycyrrhizae radix on repeated restraint stress-induced neurochemical and behavioral responses. Korean J Physiol Pharmacol 14: 371-376.

26. Kim SH, Han J, Seog DH, Chung JY, Kim N, et al. (2005) Antidepressant effect of Chaihu-Shugan-San extract and its constituents in rat models of depression. Life Sci 76: 1297-1306.

27. Pengsuparp T, Indra B, Nakagawasai O, Tadano T, Mimaki Y, et al. (2001) Pharmacological studies of geissoschizine methyl ether, isolated from Uncaria sinensis Oliv., in the central nervous system. Eur J Pharmacol 425: 211-218.

\section{Acknowledgements}

The authors declare no conflicts of interest in association with the present study. 